\title{
CARACTERISTICAS BIOCLIMATICAS DE LAS MALEZAS DEL CINTURON VERDE DE CORDOBA. Parte I - Polygonum aviculare "sanguinaria"1
}

\author{
RAUL A. NOBILE ${ }^{2}$, GRACIELA E. EDREIRA ${ }^{3}$ e JULIO DI RIENZO 4
}

\section{RESUMO}

Na região fruti-ortícula do cinturião verde da cidade de Córdoba, Argentina, ocorre com frequência nos períodos de inverno e primavera o aparecimento de Polygomun aviculare. O objetivo do presente trabalho foi o de conhecer a fenologia desta espécie e as necessidades bioclimáticas de cada uma das etapas do seu ciclo de vida. Para estas análises conduziu-se um ensaio em uma propriedade do cinturião verde, na região Nordeste de Córdoba, durante os anos de 1988, 1989 e
1990. Determinrou-se as fases e subperíodos do ciclo evolutivo da espécie e parâmetros como altura e densidade de plantas e dados meteorológicos. O estudo permitiu estabelecer o fluxo estacional de emergência e determinar o tipo ecológico de planta daninha quanto à estação de crescimento. Os aspectos ecológicos levantados servirão de ferramentas para implementar estratégias de controle desta planta daninha.

Palavras chave: Planta daninha, emergência, fenologia.

\begin{abstract}
"Knotweed", Polygonum aviculare is one of the most common weeds in the winter-spring period in the horticultural zone of Córdoba green belt. In order to know the phenology and climatic requirements of the species in each stage of its life cycle, an experiment was conducted in one of the green belt farms located in the NE of Córdoba outskirsts for three years $(1988,1989,1990)$. Different phases and sub-periods of this life cycle, phenometric parameters (plant height and density)
\end{abstract}

ABSTRACT

\section{Bioclimatic characteristic of weeds in the city Cordoba's green belt. Part I -Polygonum aviculare}

\section{INTRODUCCIÓN}

Los cultivos fruti-hortícolas del cinturón verde de la ciudad de Córdoba, presentan una diversidad de malezas latifoliadas durante las estaciones del año siendo una de ellas Polygonum and meteorological observations were determined. The experiment enabled to establish the stational flow of emergency and corroborate the winterspring ecological type of the weed. These aspects of the bioecology, phenology and bioclimatology are necessary tools when implementing an integrated system of weed control.

Key words: Weed, emergency, phenology, bioclimatology.

aviculare L. "sanguinaria o cien nudos", se trata de una hierba anual, inverno-primaveral de la familia Poligonáceas, cuyas semillas presentan dormancia tornando difícil su control (Harper, 1957).

\footnotetext{
${ }^{1}$ Recebido para publicação em 23/04/1998 e na forma revisada em 10/11/1998.

${ }^{2}$ Ing.Agr. Profesor Adjunto de Terapeútica Vegetal. Facultad de Ciencias Agropecuarias. C.C.509 - C.P.5000. Córdoba, Argentina.

${ }^{3}$ Ing.Agr. Jefe de Trabajos Prácticos de Climatología y Fenología Agrícolas. Facultad de Ciencias Agropecuarias. C.C.509 C.P.5000. Córdoba, Argentina.

${ }^{4}$ Biólogo M.Sc. Profesor Adjunto de Estadística y Biometría. Facultad de Ciencias Agropecuarias. C.C.509 - C.P.5000. Córdoba, Argentina.
} 
El estudio de "sanguinaria o cien nudos" ha sido el motivo de trabajo de varios autores, algunos de ellos basaron sus estudios en determinar la calidad de propágulos viables en suelos agrícolas; y establecer el flujo de emergencia característico de las especies, en las diferentes épocas del año. Por ejemplo Leguizamón et al., (1980), en el área de influencia de Rosario, Argentina y Roberts \& Feast, 1970, en Wellesbourne, Inglaterra .

Investigadores como Courtney, (1968); Baskin \& Baskin, (1990) estudiando semillas enterradas de Polygonum aviculare establecieron que sus semillas presentan dormancia anualestacional. Este mecanismo facilita a la maleza su persistencia en los suelos agrícolas con diferentes picos de emergencia.

De los estudios realizados se desprende que es necesario conocer la bioecología, la fenología y la bioclimatología de las malezas para poder implementar estrategias en el sistema de manejo integrado de malezas.

El objetivo del presente trabajo es conocer la fenología de la especie y los requerimientos bioclimáticos en cada una de las etapas del ciclo evolutivo en el cinturón verde de Córdoba.

\section{MATERIAL Y METODO}

El trabajo se realizó en un predio frutihortícola, ubicado en el sector Noreste del Cinturón Verde de la ciudad de Córdoba, en la localidad de Villa Esquiú a $15 \mathrm{~km}$ de la capital (Lat. Sur $31^{\circ} 19^{\prime}$ y Long. Oeste $64^{\circ} 13^{\prime}$ ).

Se trabajó durante los años 1988, 1989, 1990 en una parcela cuyo tipo de suelo es Haplustol típico de textura franco-limosa y de perfil $A_{p}, B, B_{c}, C$; sin tratamientos con fertilizantes. Se demarcaron dos surcos orientados de Este a Oeste con una extensión de $50 \mathrm{~m}$ cada uno; estos surcos recibieron igual número de riegos que los cultivos adyacentes, siendo de tres a cinco durante la estación de crecimiento y desarrollo de la maleza. Los mismos no fueron perturbados con laboreo, durante el período que estuvo la maleza.
Para realizar las mediciones meteorológicas se instaló un termohigrógrafo y un pluviómetro tipo "B" (de acuerdo a las normas de la Organización Meteorológica Mundial: OMM).

En cuanto a las observaciones fenológicas se consideraron las siguientes fenofases: emergencia, floración, fructificación y senescencia (San Martín et al., 1988), basándose la cuantificación en un método de observación fenológica para cultivos anuales, de uso común en la Argentina, descripto por Planchuelo-Ravelo et al. (1987).

Las observaciones meteorológicas se realizaron con instrumental que permitió el registro de temperatura y humedad relativa del aire de manera continua y los de precipitación con frecuencia semanal.

A partir de estos datos se obtuvieron los valores semanales mínimos, máximos y medios de temperatura, número de horas de frío, humedad relativa y precipitaciones.

Las observaciones fenológicas y fenométricas (altura de plantas, densidad de plantas) se realizaron con frecuencia semanal sobre cinco cuadrados de $0,50 \mathrm{~m}$ x $0,50 \mathrm{~m}$ localizados al azar en la parcela de estudio. Se registró el número de plantas en las diferentes fenofases y el flujo de emergencia se representó como diagrama estacional de flujo de emergencia (Roberts et al., 1970).

\section{RESULTADOS Y DISCUSION}

En los tres años de ensayo se observaron variaciones de los elementos meteorológicos, dando lugar a condiciones hídricas y térmicas diferentes que condicionaron los estados fenológicos.

Las Figuras 1, 2 y 3 muestran las curvas de evolución de temperatura media, número de horas de frío acumuladas, precipitación acumulada y humedad relativa máxima y mínima en los tres años de ensayo.

En cuanto al número de horas de frío (HF) se puede observar que la mayor acumulación corresponde al año 1988 con $1754 \mathrm{HF}$, seguido por los años 1989 con 343 HF y 1990 con 295 HF. 


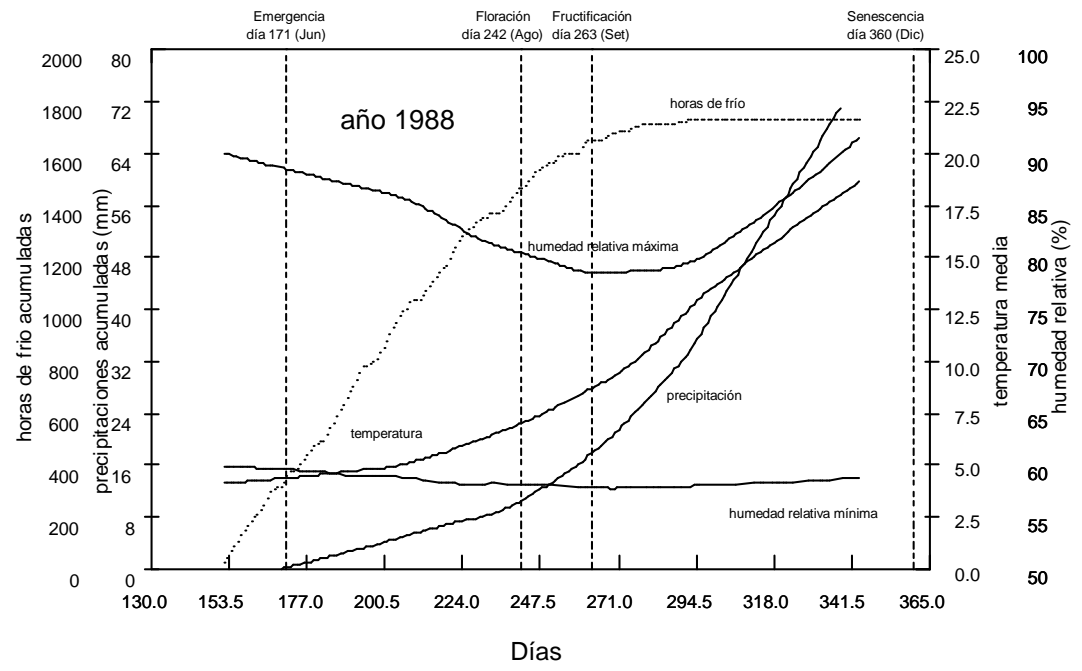

FIGURA 1. Curvas de evolución de temperatura media, número de horas de frío acumuladas, precipitación acumulada y humedad relativa máxima y mínima, durante el ciclo biológico de Polygonum aviculare, en el año 1988.

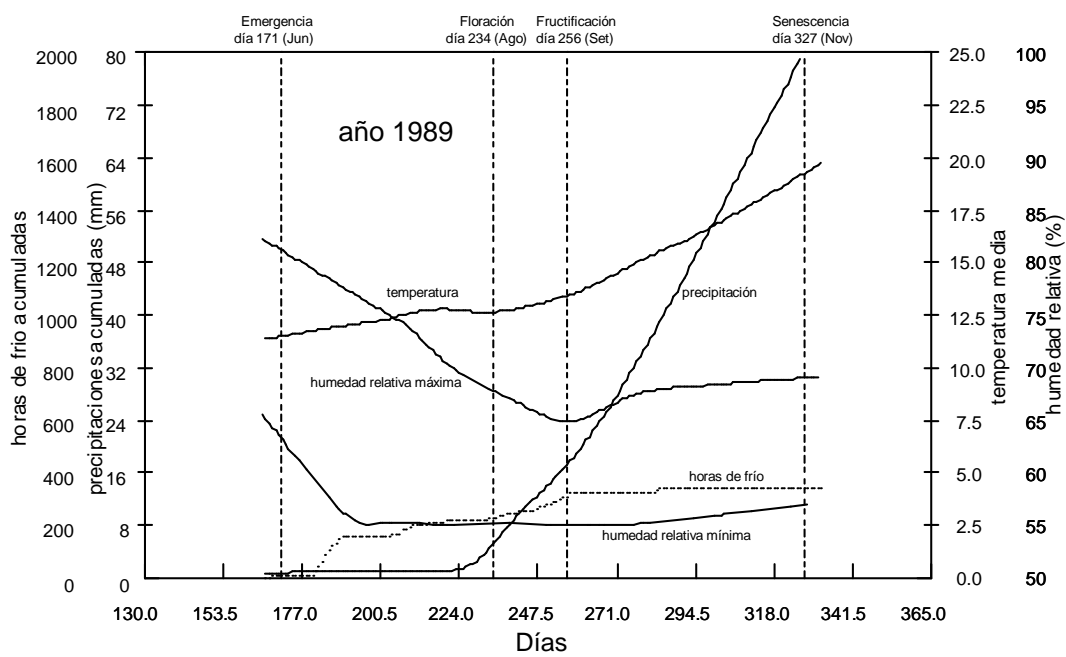

FIGURA 2. Curvas de evolución de temperatura media, número de horas de frío acumuladas, precipitación acumulada y humedad relativa máxima y mínima, durante el ciclo biológico de Polygonum aviculare, en el año 1989.

La Figura 2 permite visualizar que para el mes de Junio del año 1989 sólo se presentaron cuatro HF y para el mes de Julio del año 1988 corresponde la mayor acumulación $589 \mathrm{HF}$ (Figura 1).
Estos patrones diferenciales de acumulación del número de horas de frío expresan en parte, una mayor amplitud térmica para el año 1988 (Tabla 1). 


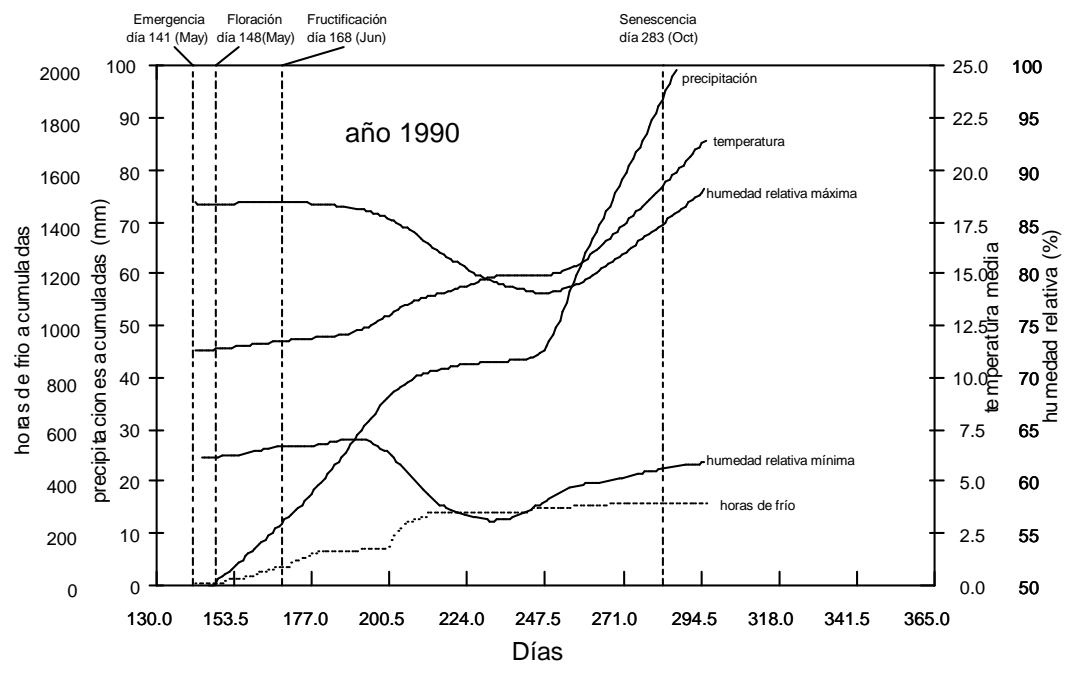

FIGURA 3. Curvas de evolución de temperatura media, número de horas de frío acumuladas, precipitación acumulada y humedad relativa máxima y mínima, durante el ciclo biológico de Polygonum aviculare, en el año 1990.

TABLA 1. Valores de temperatura media, amplitud térmica y número de horas de frío durante el ciclo de crecimiento de Polygonum aviculare L.

\begin{tabular}{ccccc}
\hline Años & $\begin{array}{c}\text { Temperatura media } \\
\text { más baja }\end{array}$ & $\begin{array}{c}\text { Temperatura } \\
\text { media más alta }\end{array}$ & Amplitud térmica & Horas de Frío \\
\hline & & & & \\
1988 & $4,8{ }^{\circ} \mathrm{C}$ & $17,1{ }^{\circ} \mathrm{C}$ & $12,3^{\circ} \mathrm{C}$ & 1754 \\
1989 & $13,0^{\circ} \mathrm{C}$ & $18,3{ }^{\circ} \mathrm{C}$ & $5,3{ }^{\circ} \mathrm{C}$ & 343 \\
1990 & $11,3{ }^{\circ} \mathrm{C}$ & $16,4^{\circ} \mathrm{C}$ & $5,1{ }^{\circ} \mathrm{C}$ & 295 \\
\hline
\end{tabular}

En lo que a precipitación respecta, el mayor monto se registró en el año 1990 con 99 mm, seguido del año 1989 con $77 \mathrm{~mm}$ y el año 1988 con $71 \mathrm{~mm}$ (Figuras 1, 2, 3). Cabe destacar que Córdoba posee un régimen de precipitación Monzónico con concentración de las lluvias en el semestre cálido: Octubre a Marzo.

Las Figuras 1 a 3 muestran las principales fases del ciclo evolutivo de la maleza, apreciándose diferencias tanto en la longitud del mismo, como en extensión de los diferentes subperíodos. El ciclo más largo corresponde al año 1988 con 189 días, seguido por los años 1989 y
1990 cuyas duraciones fueron de 156 días y 142 días respectivamente (Tabla 2).

Desde el punto de vista fenológico los años 1988 y 1989 son similares. El año 1990, en tanto, mostró diferencias en lo que respecta a la longitud del ciclo y de los subperíodos Emergencia-Floración y FructificaciónSenescencia (Tabla 2 y Figura 3), que son atribuidas a las condiciones hídricas y térmicas diferentes.

El diagrama de flujo de emergencia (Figura 4: a, b, c, ) muestra la evolución temporal de la emergencia en los tres años considerados. Aunque las fases están sincronizadas en el tiempo 
en los años 1988-1989, en este último se observa un adelanto en el pico de emergencia de aproximadamente 2 meses, ligado a una mayor concentración en la emergencia.

Un panorama diferente se presenta en el año 1990 con un pico de emergencia aún más temprano situado 3 meses y medio antes que el pico de 1988. Al igual que en el año 1989, este pico está unido a una clara concentración del período de emergencia. No obstante el pico de emergencia de mediados de Mayo, aparece un segundo pico de menor intensidad a mediados de Agosto.

TABLA 2. Longitud y diferentes subperíodos del ciclo evolutivo de Polygonum aviculare L.

\begin{tabular}{ccccc}
\hline Años & $\begin{array}{c}\text { Duración del ciclo } \\
\text { (días) }\end{array}$ & $\begin{array}{c}\text { Emergencia- } \\
\text { Floración }\end{array}$ & $\begin{array}{c}\text { Floración- } \\
\text { Fructificación }\end{array}$ & $\begin{array}{c}\text { Fructificación- } \\
\text { Senescencia }\end{array}$ \\
\hline & & & & \\
1988 & 189 & 71 & 21 & 97 \\
1989 & 156 & 63 & 22 & 71 \\
1990 & 142 & 7 & 20 & 115 \\
\hline
\end{tabular}

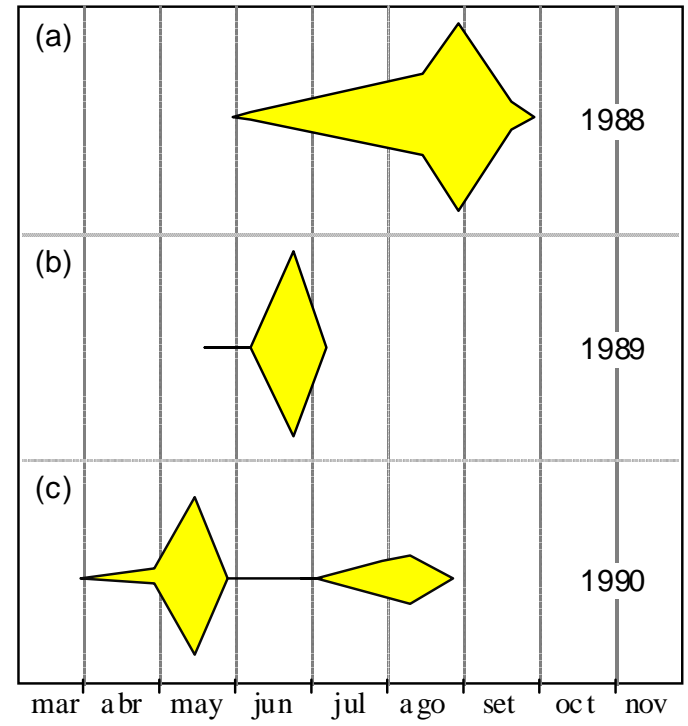

FIGURA 4. Evolución temporal de la emergencia de Polygonum aviculare en los años 1988, 1989 y 1990.

Los resultados obtenidos muestran un sensible aumento de densidad desde 1988 a 1990. Este incremento es especialmente grande del primero al segundo año, pasando de 12 plantas $/ \mathrm{m}^{2}$ a 204 plantas $/ \mathrm{m}^{2}$, esto equivale a un incremento de 17 veces.

Podemos indicar como posibles causas del aumento de la densidad, por una parte, la falta de control durante el ciclo de la maleza, y por la otra el laboreo (aradas, rastreadas, y riegos) que se realizaron durante el período estival en la rotación de la parcela, favoreciendo la redistribución de las semillas y garantizando su permanencia en el terreno, como ha sido señalado por Brenchley \& Warington, (1930); Courtney, (1968). 
"Sanguinaria" presentó un flujo estacional de emergencia concentrado en el tiempo coincidente con las observaciones de Courtney (1968) y Falcon et al. (1991). Es destacable la alta variabilidad del momento en que se manifiesta el pico de flujo de emergencia, ocurriendo desde mediados de Mayo en 1990 a fines de Agosto en 1988. Esta variabilidad, que ha sido reconocida por otros autores (Beuret, 1989; Courtney, 1968; Falcon et al., 1991; Leguizamón et al., 1980), no puede estar asociada, de acuerdo a nuestros resultados, con una única causa, aunque posiblemente las condiciones de benignidad climática de los dos últimos años del estudio, podrían ser la clave para explicar el fenómeno. No queda claro sin embargo, el papel que juegan las horas de frío como elemento indispensable para la ruptura de la dormición que ha sido señalado por varios autores para esta especie (Baskin \& Baskin, 1990; Beuret, 1989; Brenchley \& Warington, 1930; Courtney, 1968).

El análisis de las fases y subperíodos establecen el hábito de la maleza: anual e invernoprimaveral, concordando con diversas publicaciones (Falcon et al., 1991; Leguizamón et al., 1980; Lopez \& Mattiacci, 1983; Pérez, 1991; Roberts \& Feast, 1970), y contrastando con los resultados de San Martín et al. (1988) quien la considera perenne y primavero-estival quizás por la existencia de ecotipos, gran variabilidad fenotípica o error en la identificación taxonómica.

Esta contribución muestra el carácter oportunista de la maleza y la dificultad de su estudio en condiciones no controladas, cuando el objetivo es identificar los factores que regulan su ciclo y la estrategia reproductiva.

\section{BIBLIOGRAFIA}

BASKIN, J.M.; BASKIN, C.C. The role of ligth and alternating temperatures on germination of Polygonum aviculare seeds exhumed on various dates. Weed Res., v.30, p. 397-402. 1990.
BEURET, E. Influence des practiques culturales sur l'evolution de la flore adventice: etude $\mathrm{du}$ potentiel semencier des sols. Revue suisse Agric., v.21, n.2, p.75-82, 1989.

BRENCHLEY, W.E.; WARINGTON, K. The weed seed population of arable soil. I Numerical estimation of viable weeds and observation on their natural dormancy. $\mathbf{J}$. Ecol., v.18, p.235-272, 1930.

COURTNEY, A.D. seed dormancy and field emergence in Polygonum aviculare. J. App. Ecol., v.5, p. 675-684, 1968.

FALCON, L.; de BEDENDO, M.F.; de FARIAS, E.D. Flujo de emergencia de malezas. In: Malezas, Tomo 1, p. 45-52, 1991.

HARPER, J.L. The ecological significance of dormancy and its importance in weed control. Proc. $4^{\text {th }}$ int. Congr. Crop. Prot. (Hamburg), p. 415-420, 1957.

HOOP, R.J. Sistema de observación fenológica en plantas. In: Phenology and Seasonality Modeling. Edited by Helmut Lieth, 1974. P. 25-29.

KROPFF, M.J.; SPITTERS, C.J. A simple model of loss by weed competition from early observations in relative leaf area of the weeds. Weed Res., v. 31, p. 97-105, 1991.

LEGUIZAMON, E.; COLOMBO, M.E.; SALINAS, A. SEVERIN, C. Modelos de flujos de emergencia de 19 especies de malezas. Malezas, v. 8, n.2, p. 3-11, 1980.

LOPEZ, J.A.; MATTIACCI, M.R. Daño de sanguinaria (Polygonum aviculare) durante la implantación de una pastura cultivada. Malezas, Tomo 3, $2^{\circ}$ parte, v.11, n.3, p.246251, 1983. 
PEREZ, M.B. Flujo de emergencia de malezas.

Malezas, Tomo 1, p. 27-30, 1991.

PLANCHUELO-RAVELO, A.M.; RAVELO, A.C.; PASCALE, A.J. Manual del Seminario en Fenología Agrícola. Edit. INANHI. Quito, Ecuador, 1987.

ROBERTS, H.A.; FEAST, P.M. Seasonal distribution of emergence in some annual weeds. Expl. Hort., v.21, p. 36-41, 1970.

SAN MARTIN, J.; CONTRERAS, D.; RAMIREZ, C. Estudios fenológicos de malezas de Arrozales cultivados en Chile Central. Turrialba, v.38, n.1, p.23-30, 1988.

STOLLER, E.N.; WAX, L.M. Periodicity of germination and emergence of some annual weeds. Weed Sci., v.21, p.574-580, 1973. 